\title{
Simulation of marine controlled source electromagnetic measurements using a parallel fourier $h p$-finite element method
}

\author{
David Pardo • Myung Jin Nam • Carlos Torres-Verdín • \\ Michael G. Hoversten · Iñaki Garay
}

Received: 23 September 2009 / Accepted: 4 June 2010 / Published online: 9 July 2010

(C) Springer Science+Business Media B.V. 2010

\begin{abstract}
We introduce a new numerical method to simulate geophysical marine controlled source electromagnetic (CSEM) measurements for the case of 2D structures and finite $3 \mathrm{D}$ sources of electromagnetic (EM) excitation. The method of solution is based on a spatial discretization that combines a 1D Fourier transform with a 2D self-adaptive, goal-oriented, $h p$ Finite element method. It enables fast and accurate
\end{abstract}

The work reported in this paper was funded by Chevron, the Spanish Ministry of Science and Innovation under the projects TEC2007-65214, PTQ-08-03-08467, and MTM2010-16511.

D. Pardo $(\varangle) \cdot$ I. Garay

Basque Center for Applied Mathematics (BCAM),

Bilbao, Spain

e-mail: pardo@bcamath.org,dzubiaur@gmail.com

D. Pardo

IKERBASQUE, Basque Foundation for Science,

Bilbao, Spain

M. J. Nam

Department of Energy and Mineral Resources Engineering,

Sejong University, Sejong, Korea

C. Torres-Verdín

The University of Texas at Austin,

Austin, Texas, USA

M. G. Hoversten

Chevron, San Ramon, California, USA

I. Garay

Institute for Theoretical Physics III,

University of Erlangen-Nürnberg,

Erlangen-Nuremberg, Germany simulations for a variety of important, challenging and practical cases of marine CSEM acquisition. Numerical results confirm the high accuracy of the method as well as some of the main physical properties of marine CSEM measurements such as high measurement sensitivity to oil-bearing layers in the subsurface. In our model, numerical results indicate that measurements could be affected by the finite oil-bearing layer by as much as $10^{4} \%$ (relative difference). While the emphasis of this paper is on EM simulations, the method can be used to simulate different physical phenomena such as seismic measurements.

Keywords Marine controlled source electromagnetics (CSEM) • Fourier finite element method • $h p$-adaptivity $\cdot$ Goal-oriented adaptivity

\section{Introduction}

1.1 History and main principles of marine CSEM measurements

Resistivity measurements have been used during the last 80 years to quantify the spatial distribution of electrical conductivity in hydrocarbon-bearing reservoirs. Electrical conductivity is utilized to assess material properties of the subsurface, and is routinely used by oil-companies to estimate the volume of hydrocarbons (oil and gas) existing in a reservoir.

Borehole resistivity measurements have been routinely acquired by oil-companies since 1927 , when the Schlumberger brothers recorded resistivity data for the first time in Pechelbronn, France. However, marine 
CSEM measurements have only been used by oil companies in the late 1990s and early 2000s, despite the early academic research performed during the 1970s and 1980s on the topic (see [4, 6, 9]).

Marine CSEM measurements utilize a transmitter that is typically located a few meters (10-100 m) above the sea-floor and is moved by a ship (see Fig. 1). This transmitter operates at frequencies in the range of 0.25 $1.25 \mathrm{~Hz}$ in order for EM waves to reach long distances. Measurements are recorded by a set of receivers located along the sea-floor at a distance of up to $20 \mathrm{~km}$ from the source.

EM waves excited in marine CSEM travel through different materials in the path from the transmitter to the receivers. Since the media is lossy, EM waves diffuse most of their energy before arriving to the receivers. In addition, EM waves are also reflected at the interfaces between different materials (see Fig. 1, bottom panel). The signal traveling through the subsurface is the one that is useful to study the presence, location, and shape of hydrocarbon reservoirs. For that purpose, there is a need to remove the direct signals that travel trough the sea and the one reflected by the sea-air interface. Due to the low electrical conductivity of the air, the signal reflected by the air in a shallow water environment may be several orders of magnitude larger than the one traveling through the subsurface, which increases the noise-to-signal ratio, thereby increasing the difficulty of determining the presence and location of hydrocarbons within the reservoir [33].
Fig. 1 Two views of a typical marine CSEM acquisition system composed of one transmitter carried by a ship, several receivers located at the seafloor and several subsurface layers. EM waves are reflected by different material interfaces with the air and the subsurface layers (see bottom panel)

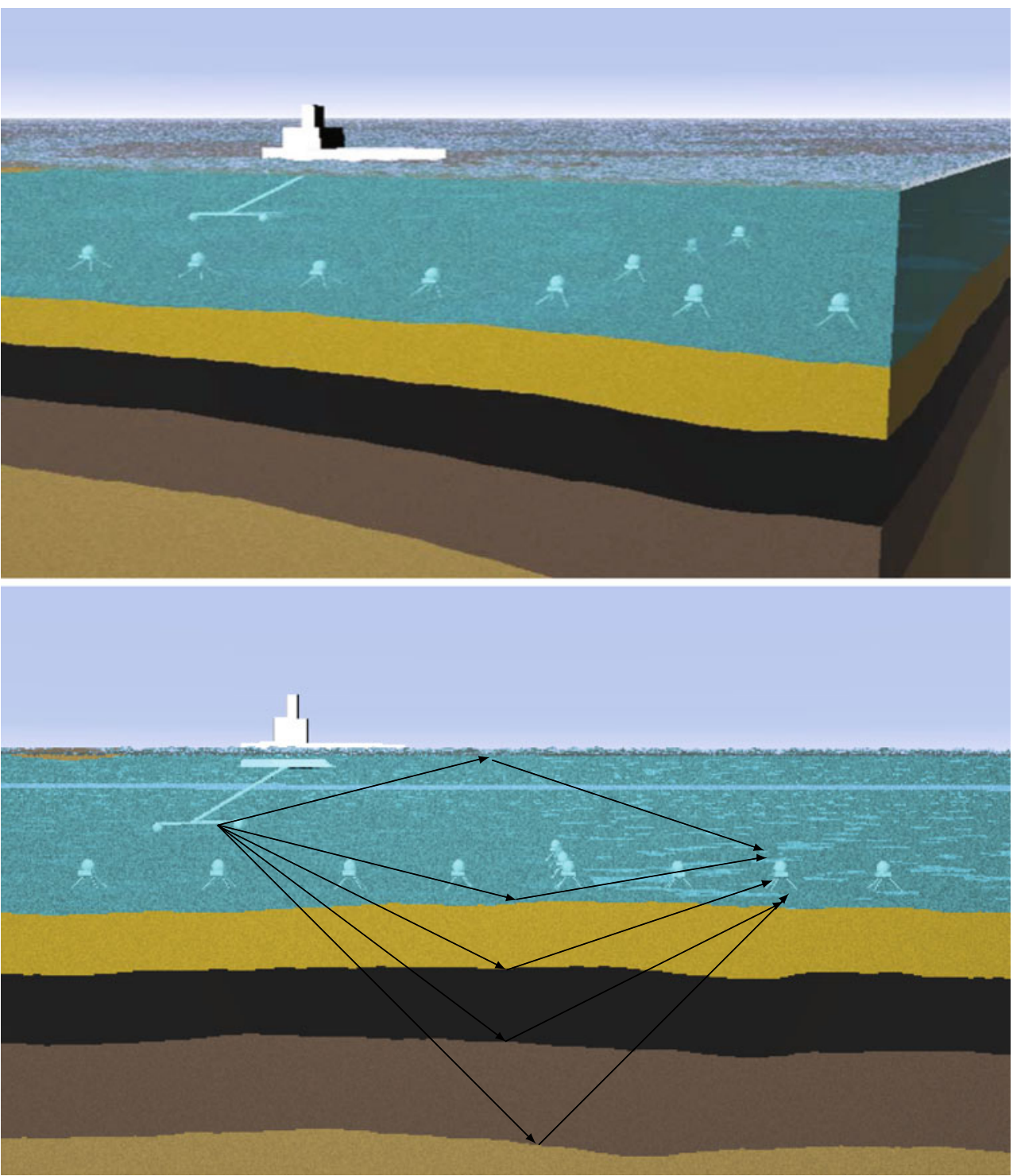


For a more detailed physical interpretation of marine CSEM measurements, see [34].

\subsection{Simulations of marine CSEM measurements}

To improve the interpretation of results obtained with resistivity measurements, and thus, to better quantify and determine existing subsurface materials and increase hydrocarbon recovery, diverse methods have been developed to perform numerical simulations (for instance, $[15,17,18,22])$ as well as to invert marine CSEM measurements [1, 5, 12, 16, 21].

Despite the existing numerical work recently developed to simulate marine CSEM measurements, several challenges need to be overcome to produce more accurate simulations in a limited amount of time. Specifically, the main numerical challenges are the following: (a) we need to consider in the same simulation objects with very different geometries and sizes, including small antennas and large subsurface layers, (b) we need to consider full EM effects, since direct current (DC) simulations at zero frequency do not provide sufficient accuracy due to the large size of the computational domain, (c) we need to consider electrically anisotropic formations, since this feature is essential to properly characterize reservoirs, (d) we need to find the solution at the receiver antennas, which is typically $5-15$ orders of magnitude smaller than the solution at the transmitter antenna, and (e) we need to produce fast simulations to enable inversion of the measurements and/or a detailed numerical study of the simulation environment.

\subsection{Fourier $h p$-Finite Element Method}

To overcome the above challenges, we propose the use of a parallel Fourier $h p$-Finite Element Method (FEM), where $h$ indicates the element size, and $p$ the polynomial order of approximation, both varying locally throughout the grid. Specifically, our method combines a (1) 2D self-adaptive goal-oriented $h p$-FEM [7, 8, $26,27]$, which provides the geometrical flexibility of a FEM as well as high-accuracy simulations due to the exponential convergence in terms of the problem size vs. the error in the quantity of interest (solution at the receiver antennas), with (1) a 1D Fourier method for considering typical scenarios of marine CSEM problems consisting of 2D subsurface resistivity models and 3D finite-size EM sources. Thus, our method is suitable for the simulation of objects with different scales (small antennas and large layers), it performs simulations at non-zero frequencies with possibly anisotropic forma- tions (we solve full Maxwell's equations), and it delivers high-accuracy approximations of the solution at the receiver antennas (quantity of interest). In addition, the entire implementation of our numerical method is compatible with the use of sequential and parallel machines in order to secure maximum performance.

The main contribution of this paper is the proper combination, extension, and application of a Fourier $h p$-Finite-Element Method [25, 30] to obtain highly accurate simulations of marine CSEM measurements. In addition, we describe a parallel implementation and compare various solvers of linear equations, we validate the method employing analytical solutions for simple marine CSEM scenarios, and illustrate via numerical experimentation the main physical principles behind marine CSEM measurements and the main features of our simulation method.

The method described in this paper provides highly accurate and reliable results within reasonable CPU times as it enables a significant reduction of the computational complexity with respect to conventional simulators without sacrifice of accuracy. In addition, the method is suitable for simulation of resistivity logging measurements, inverse problems, as well as for multiphysics applications. Therefore, it aims to become a widely used simulation strategy for problems arising in the oil industry.

The paper is organized as follows: Section 2 describes the mathematical formulation of our Fourier Finite Element Method, which we describe in detail along with the main implementation aspects on Section 3. Section 4 illustrates the performance of the method, including validation results and challenging simulations of marine CSEM measurements. Section 5 is devoted towards conclusions.

\section{Formulation}

In this section, we first introduce time-harmonic Maxwell's equations, and the corresponding modeling of the sources and boundary conditions we utilize for simulation of marine CSEM measurements. Then, we describe the corresponding 3D variational formulation. After taking a 1D Fourier transform, we derive a simple variational formulation under the assumption of constant materials in the Fourier direction. We also indicate the steps needed to obtain a $3 \mathrm{D}$ variational formulation under more general material distributions. Finally, we discuss the main differences between a variational formulation in terms of electric field $\mathbf{E}$ and magnetic field $\mathbf{H}$. 


\subsection{Time-harmonic Maxwell's equations}

Assuming a time-harmonic dependence of the form $e^{j \omega t}$, with $\omega$ denoting angular frequency, Maxwell's equations in linear media can be written as

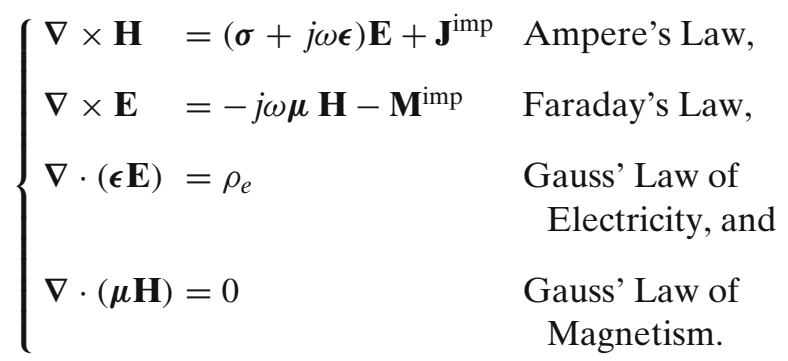

In the above equations, $\mathbf{H}$ and $\mathbf{E}$ denote the magnetic and electric fields, respectively, real-valued tensors $\boldsymbol{\epsilon}, \boldsymbol{\mu}$, and $\sigma$ stand for dielectric permittivity, magnetic permeability, and electrical conductivity of the media, respectively, $\rho_{e}$ denotes the electric charge distribution, and $\mathbf{J}^{\mathrm{imp}}, \mathbf{M}^{\mathrm{imp}}$ are representations for the prescribed, impressed electric and magnetic current sources, respectively.

For convenience, we introduce tensors $\stackrel{\circ}{\mu}:=-j \omega \mu$ and $\stackrel{\circ}{\sigma}:=\sigma+j \omega \epsilon$. We assume that $\operatorname{det}(\stackrel{\circ}{\boldsymbol{\mu}}) \neq 0$, and $\operatorname{det}(\stackrel{\circ}{\sigma}) \neq 0$.

\subsubsection{Closure of the computational domain}

A variety of BCs can be imposed on the boundary $\partial \Omega$ of a computational domain $\Omega$ such that the difference between the solution of such a problem and the solution of the original problem defined over $\mathbb{R}^{3}$ is small (see [11]). For example, it is possible to use an infinite element technique [3], a Perfectly Matched Layer (PML) [24], a boundary element technique [10] or an absorbing BC. However, it is customary in geophysical applications to impose a homogeneous Dirichlet BC on the boundary of a sufficiently large computational domain, since the EM fields decay exponentially in the presence of lossy media.

For simplicity, in the simulations presented below we will follow the Dirichlet BC approach over a 2D crosssection of the domain, that is, we will impose $\boldsymbol{n} \times \mathbf{E}=\mathbf{0}$ on the boundary of the $2 \mathrm{D}$ cross-section $\Gamma=\partial \Omega$. Specifically, we shall consider a domain of size $26 \times$ $20 \mathrm{~km}$. In the (third) spatial direction perpendicular to the plane of Fig. 1 (bottom), we will consider the original infinite domain and we will apply a Fourier transform method. We note that for the case of shallow water, we need a very large computational domain in the air layer or a more sophisticated BC (such as a PML).

\subsubsection{Source and receiver antennas}

In this work we model antennas by prescribing an impressed volume current $\mathbf{J}^{\mathrm{imp}}$ or $\mathbf{M}^{\mathrm{imp}}$. Specifically, we inject a constant horizontal electric current over a small box corresponding to the volume occupied by the electrode. Receivers measure the average electric (or magnetic) field over a small box corresponding to the volume occupied by the receiver. Notice that we do not model sources/receivers as metallic objects, but rather as small boxes with currents. We note that it is also possible to model antennas by using the equivalence principle to replace the original impressed volume currents with equivalent surface currents (see $[13,26]$ for details).

\subsection{E-variational formulation}

In this subsection, we describe our method in terms of the unknown electric field $\mathbf{E}$. First, we define the $L^{2}$ inner product of two (possibly complex- and vectorvalued) functions $\mathbf{f}$ and $\mathbf{g}$ as:

$\langle\mathbf{f}, \mathbf{g}\rangle_{L^{2}(\Omega)}=\int_{\Omega} \mathbf{f}^{*} \mathbf{g d} V$,

where $\mathbf{f}^{*}$ denotes the adjoint (conjugate transpose) of function $\mathbf{f}$.

By pre-multiplying both sides of Faraday's law by $\stackrel{\circ}{\mu}^{-1}$, multiplying the resulting equation by $\nabla \times \mathbf{F}$, where $\mathbf{F} \in H_{\Gamma}(\mathbf{c u r l} ; \Omega)=\left\{\mathbf{F} \in H(\mathbf{c u r l} ; \Omega):\left.(\mathbf{n} \times \mathbf{F})\right|_{\Gamma}=\right.$ $0\}$ is an arbitrary test function, integrating over the domain $\Omega \subset \mathbb{R}^{3}$ by parts, and applying Ampere's law, we arrive at the following variational formulation after incorporating the Dirichlet $\mathrm{BC}$ over $\Gamma=\partial \Omega$ :

$$
\left\{\begin{array}{l}
\text { Find } \mathbf{E} \in H_{\Gamma}(\mathbf{c u r l} ; \Omega) \text { such that: } \\
\left\langle\nabla \times \mathbf{F}, \stackrel{\circ}{\mu}{ }^{-1} \nabla \times \mathbf{E}\right\rangle_{L^{2}(\Omega)}-\langle\mathbf{F}, \stackrel{\circ}{\sigma} \mathbf{E}\rangle_{L^{2}(\Omega)} \\
=\left\langle\mathbf{F}, \mathbf{J}^{\mathrm{imp}}\right\rangle_{L^{2}(\Omega)} \\
\quad+\left\langle\nabla \times \mathbf{F}, \stackrel{\circ-1}{\boldsymbol{\mu}} \mathbf{M}^{\mathrm{imp}}\right\rangle_{L^{2}(\Omega)} \forall \mathbf{F} \in H_{\Gamma}(\mathbf{c u r l} ; \Omega) .
\end{array}\right.
$$




\subsubsection{Fourier transform}

We define the unitary Fourier transform of a possibly complex- and vector-valued function $\mathbf{f}\left(x_{1}\right)$ with respect to variable $x_{1}$ as follows:

$\mathcal{F}_{r}(\mathbf{f})=\mathcal{F}(\mathbf{f})(r):=\frac{1}{\sqrt{2 \pi}} \int_{\mathbb{R}} \mathbf{f}\left(x_{1}\right) e^{-j r x_{1}} \mathrm{~d} x_{1}$.

The inverse Fourier transform is given by

$\mathbf{f}\left(x_{1}\right):=\frac{1}{\sqrt{2 \pi}} \int_{\mathbb{R}} \mathcal{F}_{r}(\mathbf{f}) e^{j r x_{1}} \mathrm{~d} r$.

Two important properties of the Fourier transform are its linearity and its compatibility with differentiation, namely if $\mathbf{f}\left(x_{1}\right)$ is a differentiable function, then $\mathcal{F}_{r}\left(\frac{\partial \mathbf{f}}{\partial x_{1}}\right)=j r \mathcal{F}_{r}(\mathbf{f})$. Defining operator $\nabla^{r}:=$ $\left(j r, \frac{\partial}{\partial x_{2}}, \frac{\partial}{\partial x_{3}}\right)$, we have for $\mathbf{f}=\left(f_{1}, f_{2}, f_{3}\right)$ :

$\nabla^{r} \times\left(f_{1}, f_{2}, f_{3}\right):=\left(\frac{\partial f_{3}}{\partial x_{2}}-\frac{\partial f_{2}}{\partial x_{3}}, \frac{\partial f_{1}}{\partial x_{3}}-j r f_{3}, j r f_{2}-\frac{\partial f_{1}}{\partial x_{2}}\right)$.

Thus, we obtain the following identities:

$\mathcal{F}_{r}(\nabla \times \mathbf{f})=\nabla^{r} \times\left(\mathcal{F}_{r}(\mathbf{f})\right), \quad$ and

$\nabla \times\left(\mathbf{f}_{2 D} e^{j r x_{1}}\right)=\left(\nabla^{r} \times \mathbf{f}_{2 D}\right) e^{j r x_{1}}$,

where function $\mathbf{f}_{2 D}=\mathbf{f}_{2 D}\left(x_{2}, x_{3}\right)$ is independent of $x_{1}$.

\subsection{Fourier finite element formulation}

In this subsection, we assume that material properties (namely, $\stackrel{\circ}{\sigma}$ and $\stackrel{\circ}{\boldsymbol{\mu}}$ ) are constant with respect to $x_{1}$, and domain $\Omega$ can be expressed as $\Omega=\Omega\left(x_{1}, x_{2}, x_{3}\right)=\mathbb{R} \times$ $\Omega_{2 D}\left(x_{2}, x_{3}\right)$. Similarly, we assume $\Gamma=\mathbb{R} \times \Gamma_{1 D}\left(x_{2}, x_{3}\right)$. Using the definition of inverse Fourier transform with respect to variable $x_{1}$, we have:

$\mathbf{E}\left(x_{1}, x_{2}, x_{3}\right)=\frac{1}{\sqrt{2 \pi}} \int_{-\infty}^{\infty} \mathcal{F}_{r}(\mathbf{E})\left(x_{2}, x_{3}\right) e^{j r x_{1}} \mathrm{~d} r$.

Employing a (mono-modal) test function of the form $\mathbf{F}\left(x_{1}, x_{2}, x_{3}\right):=\frac{1}{\sqrt{2 \pi}} \mathcal{F}_{r_{m}}(\mathbf{F})\left(x_{2}, x_{3}\right) e^{j r_{m} x_{1}}$ (without the integration), we obtain:

$$
\begin{aligned}
& \langle\mathbf{F}, \stackrel{\circ}{\sigma} \mathbf{E}\rangle_{L^{2}(\Omega)} \\
& \quad=\frac{1}{\sqrt{2 \pi}}\left\langle\mathcal{F}_{r_{m}}(\mathbf{F}) e^{j r_{m} x_{1}}, \stackrel{\circ}{\sigma} \frac{1}{\sqrt{2 \pi}} \int_{\mathbb{R}} \mathcal{F}_{r_{n}}(\mathbf{E}) e^{j r_{n} x_{1}} \mathrm{~d} r_{n}\right\rangle_{L^{2}(\Omega)} .
\end{aligned}
$$

Understanding the above integral in the distributional sense, and using the fact that

$$
\frac{1}{\sqrt{2 \pi}} \int_{\mathbb{R}} e^{j r_{m} x_{1}} e^{-j r_{n} x_{1}} \mathrm{~d} x_{1}=\sqrt{2 \pi} \delta_{r_{m}, r_{n}},
$$

where $\delta_{r_{m}, r_{n}}$ is the Kronecker's delta function (equal to 1 if $r_{m}=r_{n}$ and 0 otherwise), we arrive at

$\langle\mathbf{F}, \stackrel{\circ}{\sigma} \mathbf{E}\rangle_{L^{2}(\Omega)}=\left\langle\mathcal{F}_{r_{m}}(\mathbf{F}), \stackrel{\circ}{\sigma} \mathcal{F}_{r_{m}}(\mathbf{E})\right\rangle_{L^{2}\left(\Omega_{2 D}\right)}$

Similar expressions can be obtained for all terms of the variational problem described by Eq. 3. Again, using the definition of inverse Fourier transform and Eqs. 6, 7 and 10 , we obtain:

$$
\begin{aligned}
& \langle\nabla \times \mathbf{F}, \stackrel{\circ}{\mu}-1 \nabla \times \mathbf{E}\rangle_{L^{2}(\Omega)} \\
& =\left\langle\nabla^{r_{m}} \times \mathcal{F}_{r_{m}}(\mathbf{F}), \stackrel{\circ}{\mu} \nabla^{r_{m}} \times \mathcal{F}_{r_{m}}(\mathbf{E})\right\rangle_{L^{2}\left(\Omega_{2 D}\right)}, \\
& \left\langle\mathbf{F}, \mathbf{J}^{\mathrm{imp}}\right\rangle_{L^{2}(\Omega)}=\left\langle\mathcal{F}_{r_{m}}(\mathbf{F}), \mathcal{F}_{r_{m}}\left(\mathbf{J}^{\mathrm{imp}}\right)\right\rangle_{L^{2}\left(\Omega_{2 D}\right)}, \text { and } \\
& \left\langle\nabla \times \mathbf{F}, \stackrel{\circ}{\mu}{ }^{-1} \mathbf{M}^{\mathrm{imp}}\right\rangle_{L^{2}(\Omega)}, \\
& =\left\langle\nabla^{r_{m}} \times \mathcal{F}_{r_{m}}(\mathbf{F}), \stackrel{\stackrel{\mu}{\mu}}{ } \mathcal{F}_{r_{m}}\left(\mathbf{M}^{\mathrm{imp}}\right)\right\rangle_{L^{2}\left(\Omega_{2 D}\right)} .
\end{aligned}
$$

Thus, the variational problem (3) becomes

$$
\left\{\begin{array}{l}
\text { Find } \mathbf{E}=\frac{1}{\sqrt{2 \pi}} \int_{\mathbb{R}} \mathcal{F}_{r}(\mathbf{E}) e^{j r x_{1}} \mathrm{~d} r, \text { where for each } r \in \mathbb{R}: \\
\mathcal{F}_{r}(\mathbf{E}) \in H_{\Gamma_{1 D}}\left(\mathbf{c u r l}^{r} ; \Omega_{2 D}\right), \text { and } \\
\left\langle\nabla^{r} \times \mathcal{F}_{r}(\mathbf{F}), \stackrel{\circ}{\mu}{ }^{-1} \nabla^{r} \times \mathcal{F}_{r}(\mathbf{E})\right\rangle_{L^{2}\left(\Omega_{2 D}\right)} \\
-\left\langle\mathcal{F}_{r}(\mathbf{F}), \stackrel{\circ}{\sigma} \mathcal{F}_{r}(\mathbf{E})\right\rangle_{L^{2}\left(\Omega_{2 D}\right)} \\
=\left\langle\mathcal{F}_{r}(\mathbf{F}), \stackrel{\mathcal{F}_{r}}{\left.\left(\mathbf{J}^{\mathrm{m} p}\right)\right\rangle_{L^{2}\left(\Omega_{2 D}\right)}}\right. \\
+\left\langle\nabla^{r} \times \mathcal{F}_{r}(\mathbf{F}), \stackrel{\stackrel{\mu}{\mu}}{-1} \mathcal{F}_{r}\left(\mathbf{M}^{\mathrm{imp}}\right)\right\rangle_{L^{2}\left(\Omega_{2 D}\right)} \\
\forall \mathcal{F}_{r}(\mathbf{F}) \in H_{\Gamma_{1 D}}\left(\mathbf{c u r l}^{r} ; \Omega_{2 D}\right),
\end{array}\right.
$$

where

$$
\begin{aligned}
& H_{\Gamma_{1 D}}\left(\operatorname{curr}^{r} ; \Omega_{2 D}\right) \\
& \quad=\left\{\mathcal{F}_{r}(\mathbf{F}) \in H\left(\operatorname{curr}^{r} ; \Omega_{2 D}\right):\left.\left(\mathbf{n} \times \mathcal{F}_{r}(\mathbf{F})\right)\right|_{\Gamma_{1 D}}=\mathbf{0}\right\}, \\
& H\left(\operatorname{curl}^{r} ; \Omega_{2 D}\right) \\
& \quad=\left\{\mathcal{F}_{r}(\mathbf{F}) \in \boldsymbol{L}^{2}\left(\Omega_{2 D}\right): \nabla^{r} \times \mathcal{F}_{r}(\mathbf{F}) \in \boldsymbol{L}^{2}\left(\Omega_{2 D}\right)\right\} .
\end{aligned}
$$

Variational Formulation (13) is composed of a sequence of independent $2 \mathrm{D}$ problems, a so-called 2.5D problem. The unknowns of each $2 \mathrm{D}$ problem are the three components of the electric field. Each component of the electric field depends upon two spatial variables, namely, $x_{2}$ and $x_{3}$. To derive Formulation (13), we have assumed that material properties $(\stackrel{\circ}{\sigma}$ and $\stackrel{\circ}{\mu})$ are constant with respect to variable $x_{1}$. However, we 
remark that material properties may incorporate any type of anisotropy. This is a typical requirement for the simulation of marine CSEM measurements.

\subsubsection{D Fourier finite element formulation}

For the case of general 3D materials, Eq. 11 and subsequent formulas no longer hold, and it is necessary to perform a Fourier transform of the material coefficients. As a result, the final variational formulation is expressed as a sequence of coupled 2D problems. Specifically, each 2D problem couples with as many 2D problems as terms are needed to describe exactly the material coefficients in the Fourier transform.

\subsection{H-variational formulation}

To obtain a formulation in terms of magnetic field $\mathbf{H}$ rather than in terms of electric field $\mathbf{E}$, it is sufficient to follow a similar approach to the one described in this paper but by taking as the main equation the curl of Faraday's law rather than the curl of Ampere's law.

In this work, we have implemented both formulations for comparison purposes. We note that BCs should be consistent in order to compare results obtained with the E-formulation and the $\mathrm{H}$-formulation. Specifically, the Dirichlet BCs used with the Eformulation in order to truncate the computational domain become Neumann BCs for the case of the Hformulation. Thus, the problem becomes singular (no Dirichlet BC is present), and a Dirichlet node should be introduced, as described in [7].

The simultaneous use of both E- and H-formulations could be used for error analysis of the discretization error or the truncation error if we use non-consistent BC's. A large discrepancy among both solutions indicates a large error, although unfortunately, the opposite is not true in general.

\section{Method: a Fourier $h p$-Finite Element Method}

To solve variational problem (13), we first approximate the inverse Fourier transform of electric field $\mathbf{E}$ by the following Fourier series:

$$
\begin{aligned}
\sqrt{2 \pi} \mathbf{E}(x) & =\int_{\mathbb{R}} \mathcal{F}_{r}(\mathbf{E}) e^{j r x} \mathrm{~d} r \approx \sum_{n=-M}^{M}\left(r_{n+1}-r_{n}\right) \mathcal{F}_{r_{n}}(\mathbf{E}) e^{j r_{n} x} \\
& \approx \frac{2 \pi}{T} \sum_{n=-M}^{M} \mathcal{F}_{2 \pi n / T}(\mathbf{E}) e^{j x 2 \pi n / T}
\end{aligned}
$$

In the above approximations, we have selected an integration rule with all weights equal to 1 , we have truncated the infinite sum, and we have considered a uniform distance between integration points. This distance is determined by parameter $T$. We note that as $M \longrightarrow \infty$, the number of terms and computational cost needed to properly approximate the above Fourier series expansion increases. It is also well-known that Fourier series expansions are used to represent periodic functions. Thus, a finite value of $T$ can be physically interpreted as the presence of infinite sources at distances $T, 2 T, 3 T$, etc. from the original source along the Fourier direction. These additional undesired sources may decrease the accuracy of the solution for small $T$.

To select a first approximation of $T$ that can be later adjusted based on the numerical results, we first assume that the solution decays as $\left(4 \pi \sigma d^{3}\right)^{-1}$, where $d$ is the distance from the source to the receiver. This formula corresponds to the exact solution of a point source operating at zero frequency in a homogeneous medium. Under this assumption, we conclude that

$$
\begin{aligned}
& \text { Rel. Error (in \%) } \\
& =100 \cdot\left|\sum_{n=-\infty}^{n=\infty}\left[\frac{1}{4 \pi \sigma\left((n T)^{2}+d^{2}\right)^{3 / 2}}\right]-\frac{1}{4 \pi \sigma d^{3}}\right| \\
& =\left|\sum_{n=-\infty, n \neq 0}^{n=\infty} \frac{100}{\left((n T / d)^{2}+1\right)^{3 / 2}}\right| \\
& =\left|\sum_{n=1}^{n=\infty} \frac{200}{\left(1+(n \alpha)^{2}\right)^{3 / 2}}\right|,
\end{aligned}
$$

where $\alpha=T / d$. Table 1 describes the behavior of the above formula. We observe relative errors below $25 \%$ and $10 \%$ for values of $\alpha$ equal to $2(T=2 d)$ and $3(T=3 d)$, respectively. An error level below $10 \%$ is acceptable and corresponds to what it is considered an "accurate" solution in the context of marine CSEM measurements (note that a relative difference below $10 \%$ in Fig. 4 would be almost invisible to the human eye).

After selecting $T$, we truncate the infinite series (15). An approximation of the truncation error is numerically evaluated by computing two (or four) additional terms in the Fourier series expansion.

Each term $\mathcal{F}_{r}(\mathbf{E})$ of the infinite Fourier series expansion (15) is computed using a 2D self-adaptive, goal-oriented, $h p$-FEM software that accurately simulates problems involving coupled $H$ (curl)- and $H^{1}$ discretizations. For simplicity, we construct a unique common $h p$-grid for all Fourier modes based on the 
Table 1 Relative error (in percentage) according to Eq. 16 due to the use of a non-zero value of $\alpha=T / d$

\begin{tabular}{lrrrrrrrr}
\hline Values of $\alpha$ & 1 & 2 & 3 & 4 & 6 & 10 & 100 & $1 . \mathrm{e} 3$ \\
Rel. error (in \%) & 102 & 23 & 8 & 3.5 & 1.8 & $2.4 \mathrm{e}-1$ & $2.4 \mathrm{e}-4$ & $2.4 \mathrm{e}-7$ \\
\hline
\end{tabular}

solution of the central Fourier mode. The goal-oriented $h p$-FEM delivers exponential convergence rates in terms of the error in the quantity of interest versus the number of unknowns and CPU time. The outstanding performance of the $h p$-FEM for simulating diverse resistivity logging measurements has been documented in $[25,28,29]$, and a similar performance is expected to hold when simulating marine CSEM measurements. A detailed description of the $h p$-FE method and its exponential convergence properties can be found in [7]. We refer to [26] for technical details on the goaloriented adaptive algorithm applied to simulation of electrodynamic problems.

\subsection{Solver of linear equations}

The choice of an adequate solver of linear equations is critical for the efficient simulation of marine CSEM measurements. While iterative solvers are faster and utilize less memory than direct solvers (see Table 2), they also include a number of disadvantages. Namely, when solving a problem with $M>1$ right-hand sides (as it occurs in inverse problems), the cost of iterative solvers rapidly increases, while the cost of direct solvers remains almost constant if $M<<N$. In addition, convergence properties of iterative solvers are highly dependent upon the condition number of the matrix and the physics of the problem (e.g., specific iterative solvers are needed for EM problems in order to avoid spurious modes). In particular, an iterative solver requires the implementation of smoothers specially designed to minimize the error of both the rotational and gradient parts of the solution, c.f. [2, 14]. For simplicity, and in order to avoid additional numerical errors possibly introduced by the iterative solver, in this paper we use a direct solver.

In the following, we compare the performance of two well known direct solvers of linear equations when applied to a marine CSEM problem: 1) the parallel multi-frontal solver MUMPS (version 4.8.3) [20]; and 2) PARDISO [23]. Both solvers have been combined with the ordering of the unknowns provided by METIS (version 4.0) [19].

Figure 2 compares the performance-CPU time (left panel) and random access memory (RAM) (right panel) - of the sequential versions of PARDISO and MUMPS using both the in-core and out-of-core versions of the solvers, when applied to the problem described in Fig. 3. Results indicate that the out-ofcore version of PARDISO is not competitive (utilizes roughly twice as more RAM and is twice slowest than MUMPS out-of-core). Although the in-core version of PARDISO is the fastest of all, in this work we utilize the out-of-core version of MUMPS due to its low memory requirements and the possibility of using it in distributed memory parallel machines (PARDISO only supports shared memory parallel machines). We note that in Fig. 2 we could not solve the problem with 3.2 million unknowns using PARDISO, because the solver stopped with an error indicating the lack of available RAM.

Different performance of direct solvers can be due to implementation aspects and/or better ordering of the unknowns. In the case of solvers PARDISO and MUMPS, both solvers use the ordering of unknowns provided by METIS. Thus, it seems that in-core PARDISO is able to handle zeros in a more efficient way (reason why we observe less memory consumption), while the out-of-core implementation is clearly less efficient than the one performed by MUMPS. Finally, we emphasize that due to the similar structure of the stiffness matrix for the marine CSEM problems considered in this paper, we have observed almost identical results for various CSEM problems in terms of performance of direct solvers, which we have omitted in this paper to avoid repetition.

Table 2 Scalability of direct vs. iterative solvers

\begin{tabular}{lll}
\hline Scalability & Direct solver (M right-hand sides) & Iterative solver (M right-hand sides) \\
CPU Time & $\mathrm{O}\left(N^{2} b\right)+\mathrm{O}(N M b)$ LU fact. + Backward Subst. & $\mathrm{O}\left(n^{2} b\right)+\mathrm{O}(M N b c)$ Preconditioner + Iter. Solution \\
Memory & $\mathrm{O}(N s b)$ & $\mathrm{O}(N b)$ \\
\hline
\end{tabular}

$N$ is the problem size, $b$ is the (average) bandwidth of the matrix, $c$ is a constant that depends upon the condition number of the matrix and the choice of preconditioner, $n$ is the size of the preconditioner, $s$ is a constant that depends upon the structure of the matrix, and can be as large as $N$, and $M$ is the number of right-hand sides 


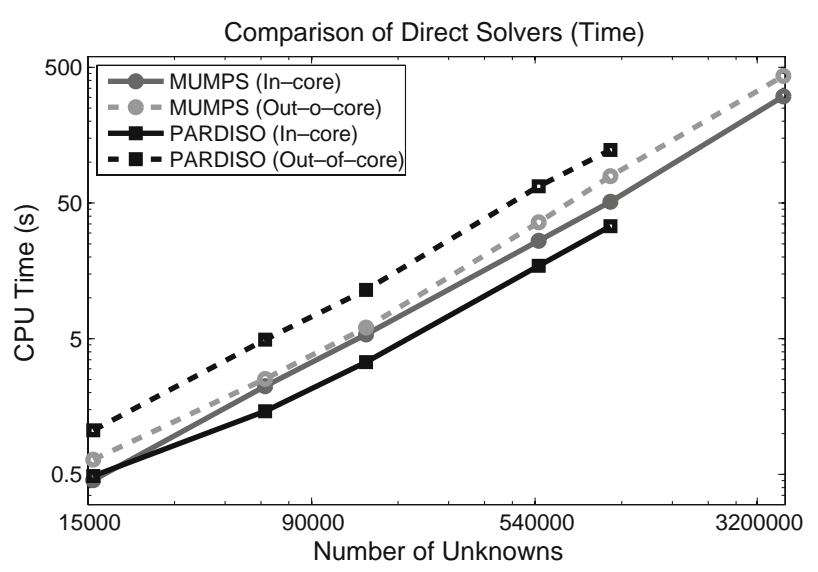

Fig. 2 CPU time (left panel) and memory (right panel) used by different direct solvers (analysis and LU factorization) vs. problem size when applied to a marine CSEM problem using

\subsection{Parallel implementation}

Finally, we implement a parallel distributed-memory version of our $h p$-FEM to speedup computations and minimize the CPU time and memory used per processor. Contrary to traditional parallelization strategies based on domain-decomposition schemes (em e.g., [31]), in our implementation we store a copy of the entire computational grid in all processors. Decision about grid refinements is performed in parallel, while actual refinements are executed in all processors simultaneously, making unnecessary the so-called gridreconciliation step intended to ensure the compatibility of refinements occurring in different processors. Thus, the complexity of the parallel version of the software is drastically simplified with respect to more traditional parallelization strategies, while the overhead associated with storing an entire copy of the $2 \mathrm{D}$ grid in all

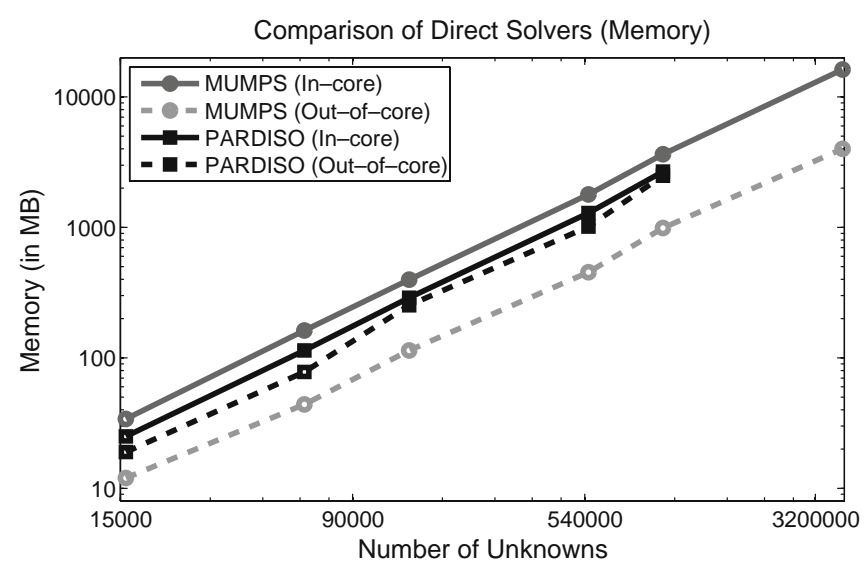

a Fourier $h p$-Finite Element Method. Tests performed on a computer equipped with $32 \mathrm{~GB}$ of RAM and using only one core of the available $2 \mathrm{GHz}$ dual-core processor

processors remains at a level below $10 \%$ of the total memory and CPU time cost for a moderate number of processors (below 200).

For the marine CSEM problems considered in this paper, we observe an adequate performance up to a moderate number of processors (16-64). Then, as we further increase the number of processors, a more rapid deterioration on the performance occurs due to the overhead associated with our simple parallelization strategy.

\section{Numerical results}

In this section, we first verify our results by comparing them against a semi-analytical software for a typical but simple marine CSEM model. Then, we perform more
Fig. 3 Marine CSEM problem composed of: a an air layer, b a 1,000-m thick layer of sea-water with resistivity equal to $0.3 \Omega \cdot \mathrm{m}$, c a background material with resistivity equal to $1 \Omega \cdot \mathrm{m}$, d a 100 -m thick (infinite lateral extend) oil-saturated layer with resistivity equal to $100 \Omega \cdot m$. . e a transmitter (horizontal electric dipole) and $\mathbf{f}$ ten equally spaced receivers. Distance between transmitter and receivers: from 1,000 to $10,000 \mathrm{~m}$

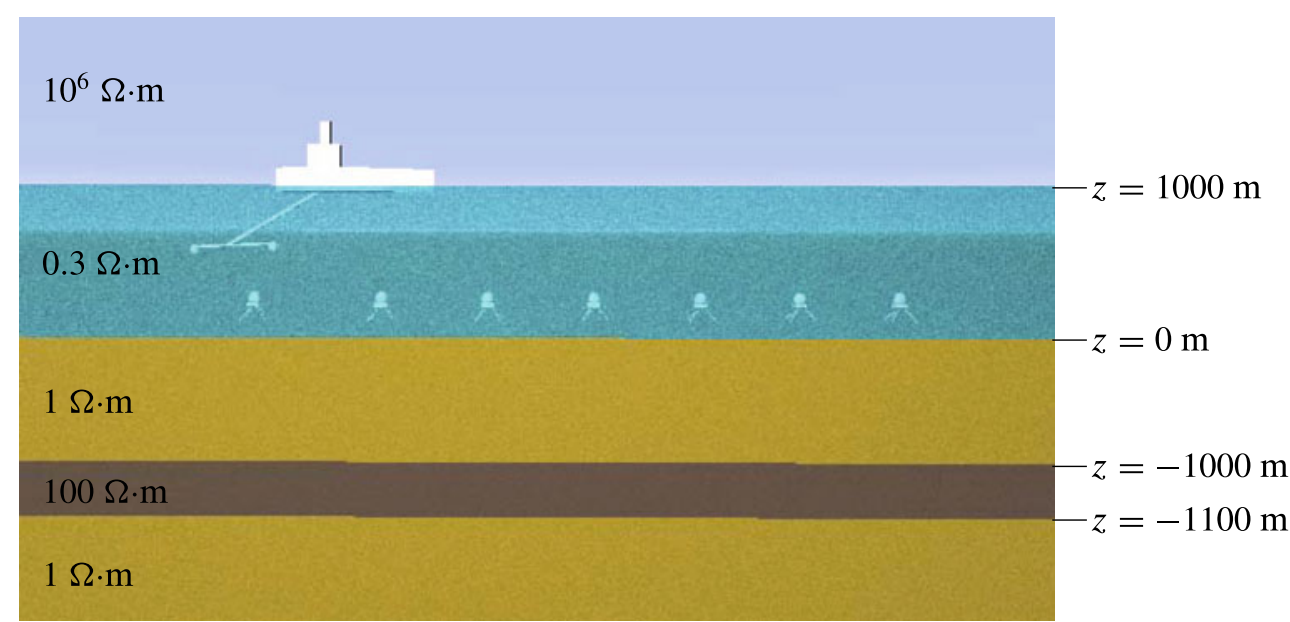


challenging numerical simulations, which enables an understanding of the main physical principles governing marine CSEM measurements. In all our models we assume that the relative magnetic permeability $\mu_{0}$ and relative permittivity $\epsilon_{0}$ are equal to 1 over the entire domain.
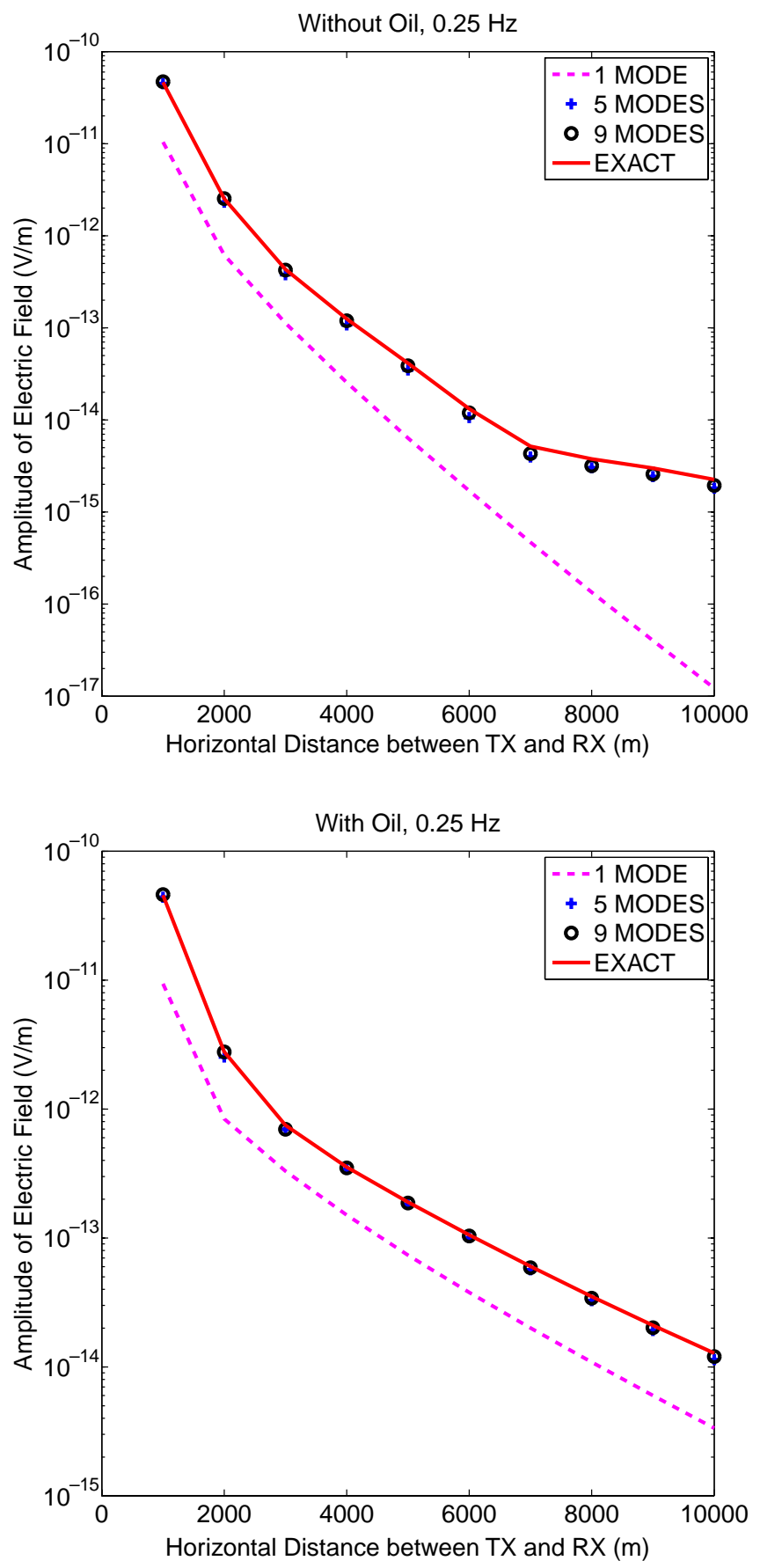

Fig. 4 Amplitude of the electric field as a function of the horizontal distance between transmitter and receivers. Different curves indicate different numbers of Fourier modes: a one mode (dotted

\subsection{Verification}

As illustrated in Fig. 3, we consider a marine CSEM problem composed of a $100 \mathrm{~m}$-thick (infinite extend) oil-bearing layer in a background subsurface material, a 1,000-m thick layer of water, a transmitter located
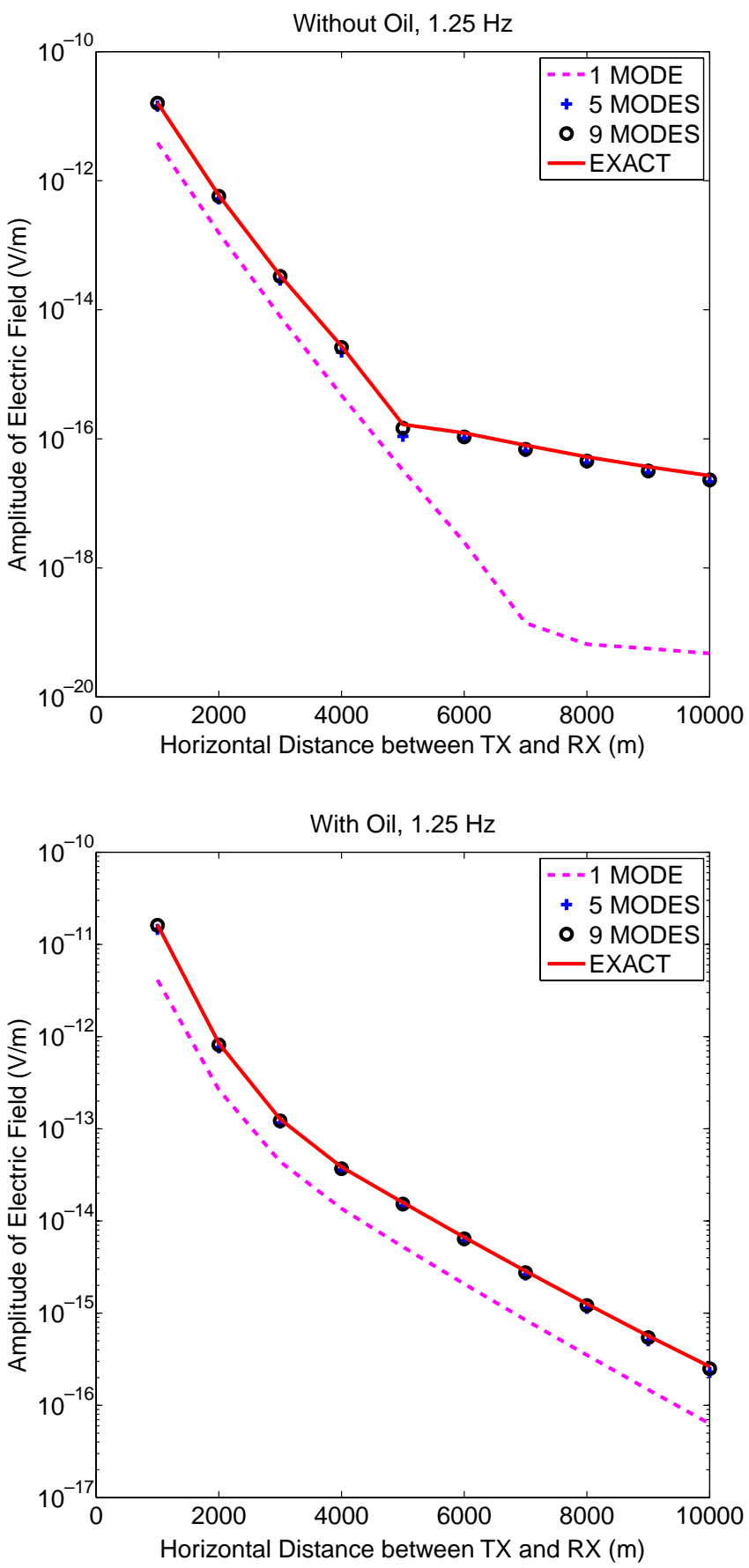

pink), b five modes (blue '+'), c nine modes (black circles), and d exact solution (red solid line). Operating frequencies: $0.25 \mathrm{~Hz}$ (left panel) and $1.25 \mathrm{~Hz}$ (right panel) 


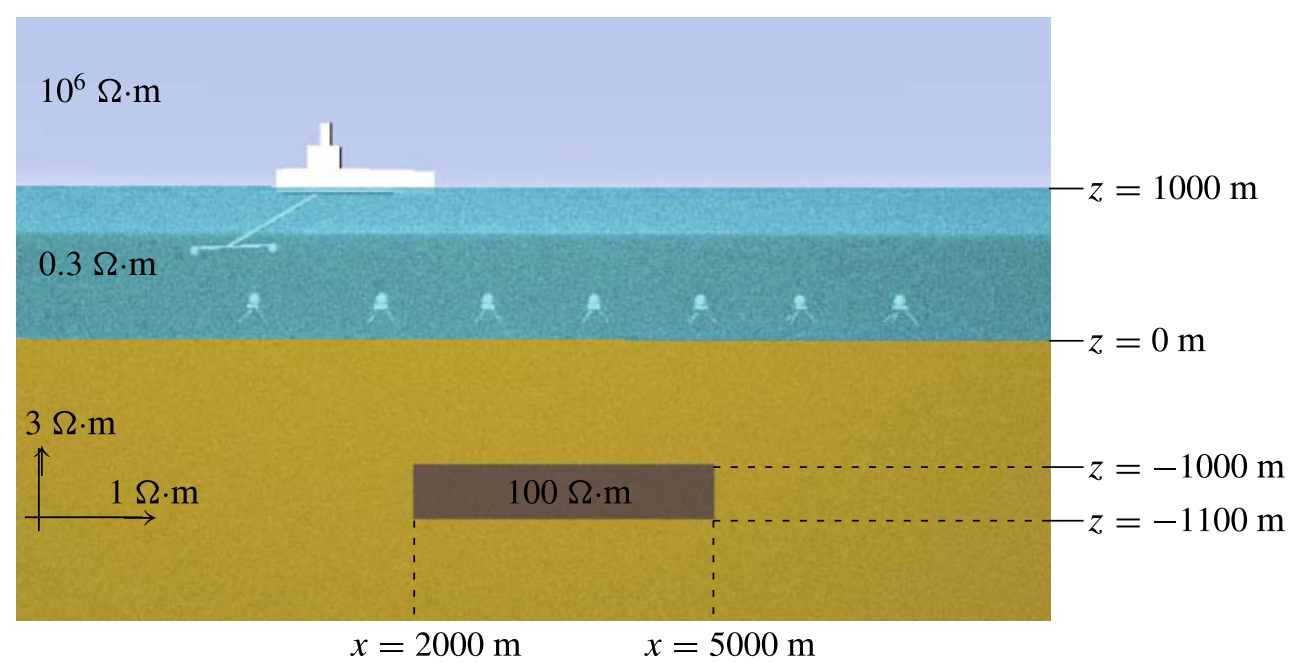

Fig. 5 Marine CSEM problem composed of: a an air layer, b a $1,000-\mathrm{m}$ thick layer of sea-water with resistivity equal to $0.3 \Omega \cdot \mathrm{m}$, c a background material with anisotropic resistivity $(1 \Omega \cdot \mathrm{m}$ in the horizontal direction and $3 \Omega \cdot \mathrm{m}$ in the vertical direction), $\mathbf{d}$ a 100-m thick (3,000-m lateral extend) oil-saturated layer with resistivity equal to $100 \Omega \cdot \mathrm{m}$., e a transmitter (horizontal electric dipole) and $\mathbf{f}$ ten equally spaced receivers. Distance between transmitter and receivers: from 1,000 to $10,000 \mathrm{~m}$
$50 \mathrm{~m}$ above the sea-floor, and ten equally spaced receivers located on the sea-floor at horizontal distances varying from 1,000 to $10,000 \mathrm{~m}$.
Figure 4 displays the amplitude of the electric field as a function of the horizontal distance between transmitter and receivers for the model problem of Fig. 3

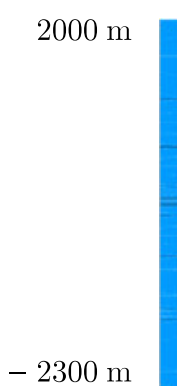

$-2500 \mathrm{~m}$

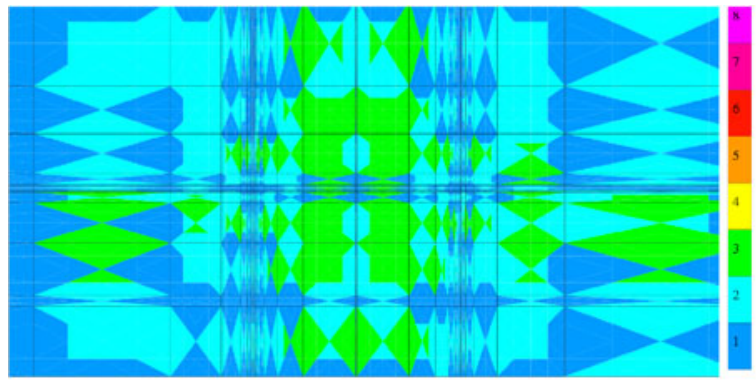

$4700 \mathrm{~m}$
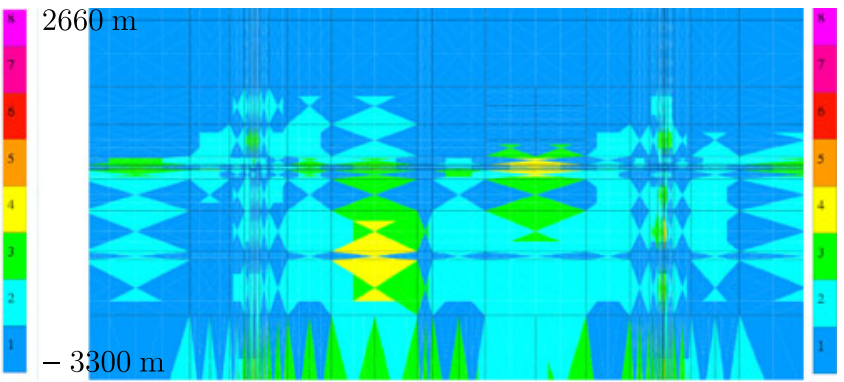

$-2300 \mathrm{~m}$

$7000 \mathrm{~m}$

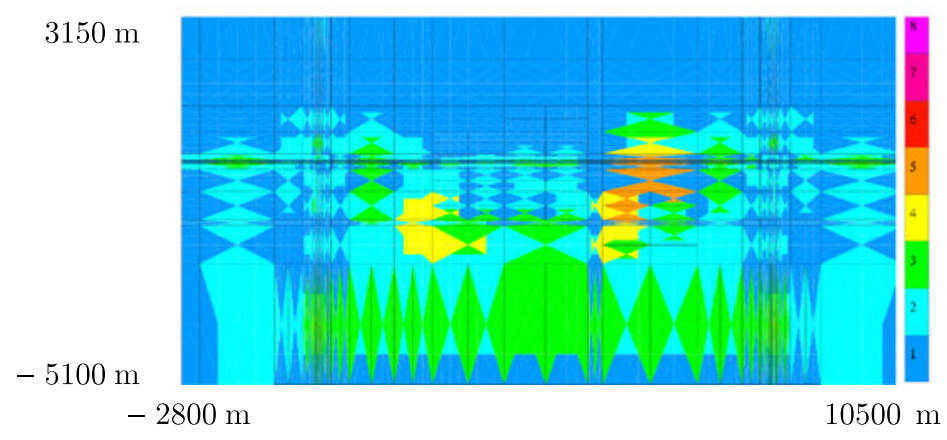

Fig. 6 Final $h p$-grid for our model problem, assuming a lateral extent of the oil-saturated layer equal to $3,000 \mathrm{~m}$, starting at a horizontal distance of 2,000 $\mathrm{m}$ away from the transmitter, and ending at 5,000 $\mathrm{m}$ away from the transmitter. Different panels correspond to different receiver locations, since we have constructed one optimal $h p$-grid for each receiver location. Specifically, we display three grids corresponding to three receivers located at horizontal distances equal to 2,000 m (top-left), 5,000 m (topright), and $8,000 \mathrm{~m}$ (bottom) away from the transmitter. Different colors indicate different polynomials orders of approximation, ranging from $p=1$ to $p=8$ 
(with and without the oil-saturated layer). The exact solution for this problem has been computed using the analytical software EM1D (authored by Professor K.H. Lee, Lawrence Berkeley Labs, Personal Communication, 2007) [32]. Numerical and analytical results coincide at both 0.25 and $1.25 \mathrm{~Hz}$. Indeed, when using only five Fourier modes, the numerical solution and the exact solution already coincide for all practical purposes.

From the physical point of view, we observe the expected behavior that the decay of the solution becomes more pronounced as frequency increases. We also observe in Fig. 4 (top panel) the influence of the waterair interface in the response, which can be noticed at distances above 7,000 and 5,000 $\mathrm{m}$ at 0.25 and $1.25 \mathrm{~Hz}$, respectively. In addition, results of Fig. 4 (bottom panel) clearly indicate the presence of oil-bearing resistive layer, because the intensity of the electric field at the receivers is larger than for the model without the oil-bearing layer (top panel).

\subsection{Numerical simulations}

Now, as described in Fig. 5, we consider a finite oil-bearing layer embedded in a possibly anisotropic formation.

In order to illustrate the optimal 2D grids that are automatically generated by the self-adaptive goaloriented $h p$-FEM, we display in Fig. 6 the final $h p$-grids delivering an error in the quantity of interest below $1 \%$ for the model problem described in Fig. 5 assuming a formation with an isotropic resistivity equal to $1 \Omega \cdot \mathrm{m}$. We emphasize that a $1 \%$ relative error in the quantity of interest provides a highly accurate solution for the case of marine CSEM problems, since EM fields suffer
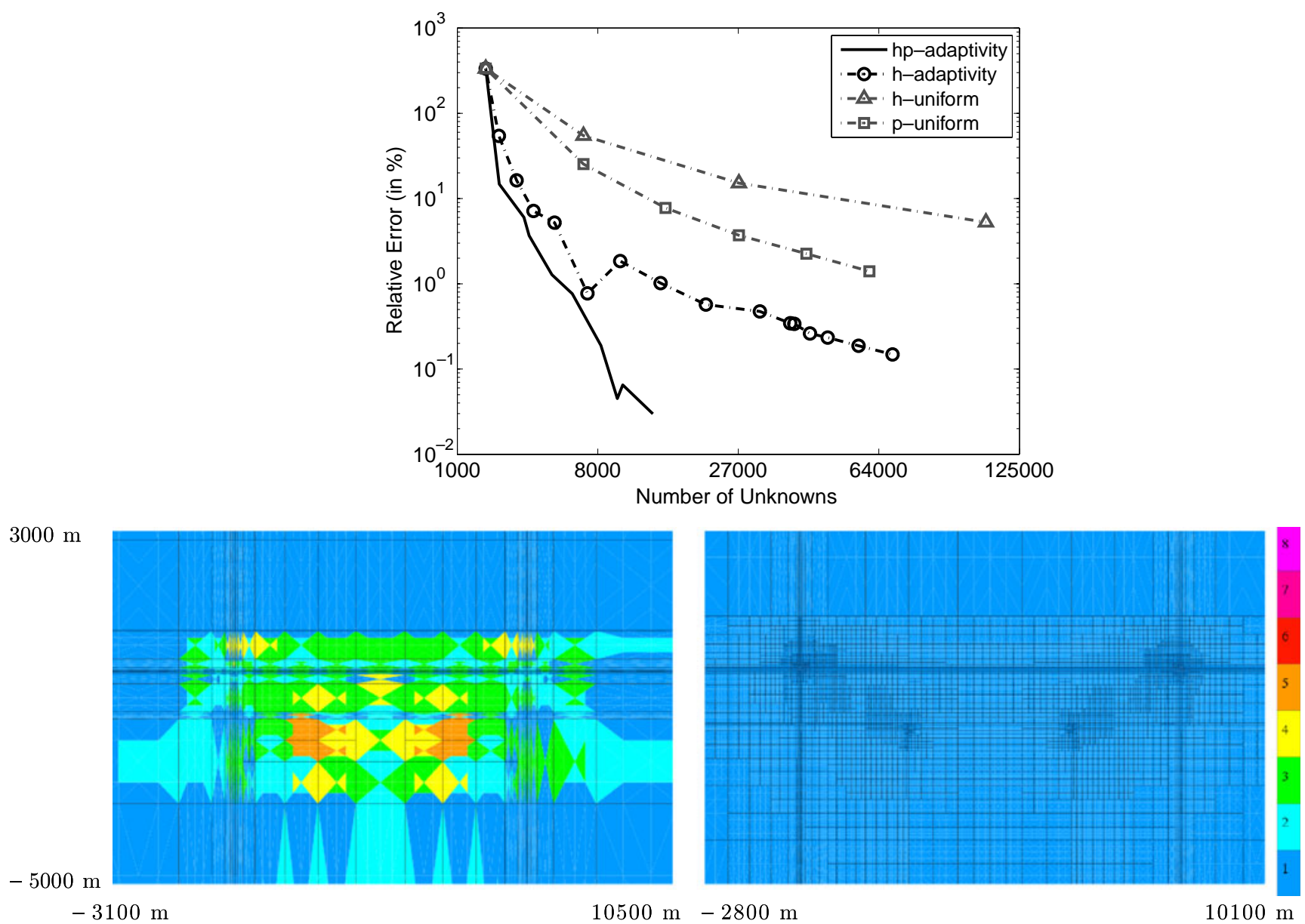

Fig. 7 Top panel: Convergence history of $h$-uniform, $p$-uniform, goal-oriented $h$-adaptive, and goal-oriented $h p$-adaptive refinements for the central Fourier mode. Bottom panel: Final goaloriented $h$-and $h p$-adaptive grids delivering $1 \%$ relative error in the solution at the receiver, which is located 7,000 $\mathrm{m}$ away from the transmitter. Different colors indicate different polynomials orders of approximation, ranging from $p=1$ to $p=8$ 
large attenuation as they travel from the transmitter to the receivers.

Our goal-oriented grid-refinement strategy is based on minimizing the error of the solution at the receiver antennas by using the dual (adjoint) solution. We observe in Fig. 6 that most $h$-refinements occur around both the transmitter and receiver electrodes, while most $p$-refinements take place in the subsurface. These refinements are consistent with the physics of the problem, since the solution is known to be smooth on the subsurface, with the most abrupt variations occurring around the transmitter and receivers electrodes. We remark that since we only consider structured initial $h p$-grids, there exist small elements used to reproduce the geometry of sources and receivers that still remain in the final $h p$-grid. However, we also observe new elements arising from local $h p$-refinements that are needed in order to reduce the discretization error.

Figure 7 (top panel) describes the convergence history of $h$-uniform, $p$-uniform, goal-oriented $h$-adaptive, and goal-oriented $h p$-adaptive refinements for the central Fourier mode when applied to the marine CSEM problem described in Fig. 5 with the receiver located
$7,000 \mathrm{~m}$ away from the transmitter and operating at $0.75 \mathrm{~Hz}$. We observe a dramatically faster convergence when using $h p$-adaptive finite element methods. The corresponding final goal-oriented $h$ - and $h p$-adaptive grids delivering $1 \%$ relative error in the solution at the receivers are displayed in Fig. 7 (bottom panel).

Figure 8 (top panel) describes the vertical component of the magnetic field $\left(H_{z}\right)$ for the direct (top-left panel) and dual (top-right) problems corresponding to the marine CSEM problem described in Fig. 5 with the receiver located $7,000 \mathrm{~m}$ away from the transmitter. Figure 8 (bottom panel) describes $H_{z}$ of the direct solution multiplied by $H_{z}$ of the dual solution, a quantity used to determine the Jacobian matrix needed for inversion.

Finally, Fig. 9 compares results for four different models: (a) the model of Fig. 3 without the oil-bearing layer, (b) the model of Fig. 3 (with an oil-bearing layer), (c) the model of Fig. 5 without anisotropy, and (a) the model of Fig. 5 (with anisotropy).

From the results shown in Fig. 9 we draw the following physical conclusions: First, it is essential to consider the effect of anisotropy in the formation because

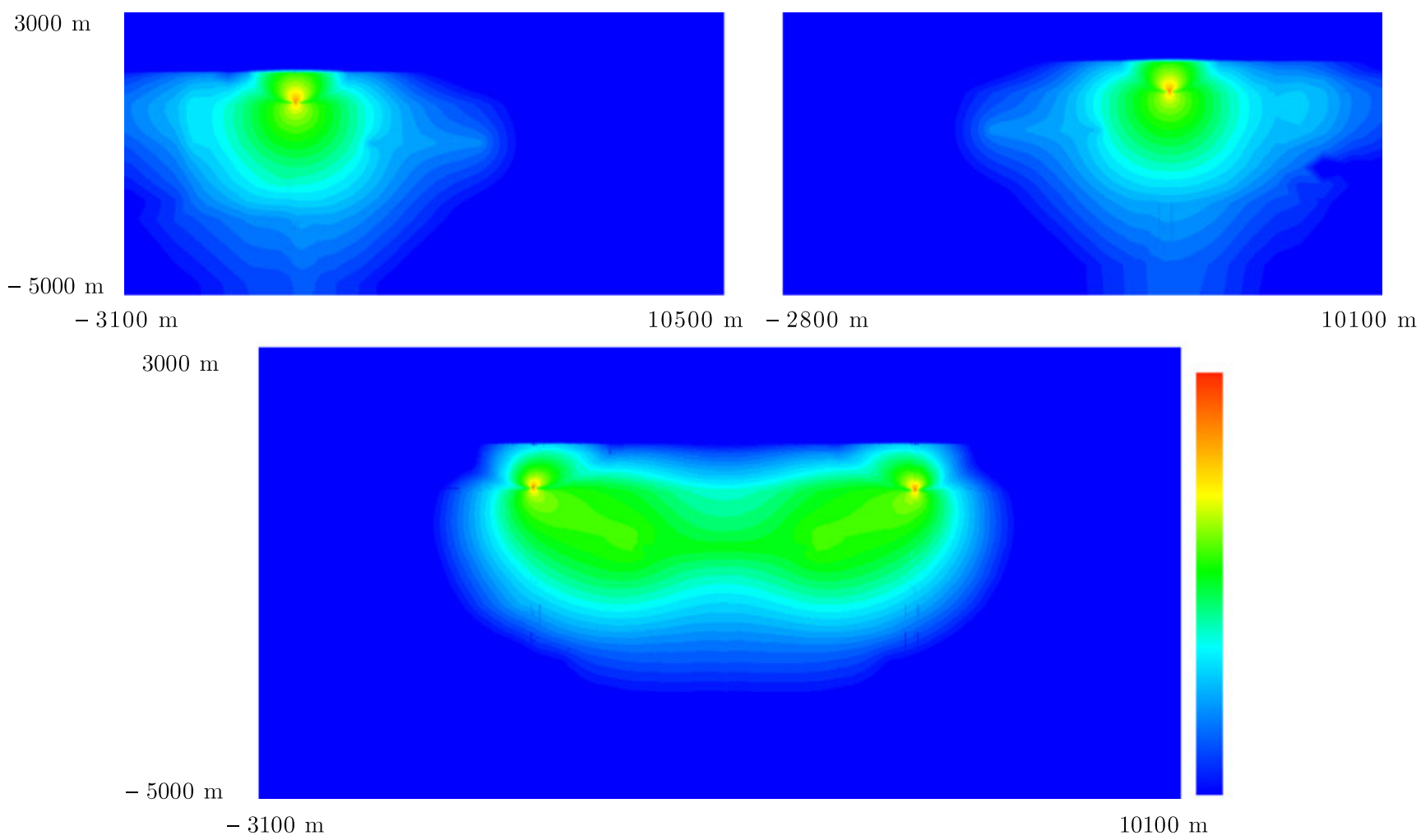

Fig. 8 Top panel: Vertical component of $H_{z}$ for the direct (topleft panel) and dual (top-right) problems corresponding to the marine CSEM problem described in Fig. 5 with the receiver located 7,000 m away from the transmitter. Bottom panel: $H_{z}$ for the direct problem multiplied by $H_{z}$ for the dual problem, which is a quantity used to compute the Jacobian matrix needed for gradient-based inversion methods. All the above quantities are displayed in logarithmic scale 

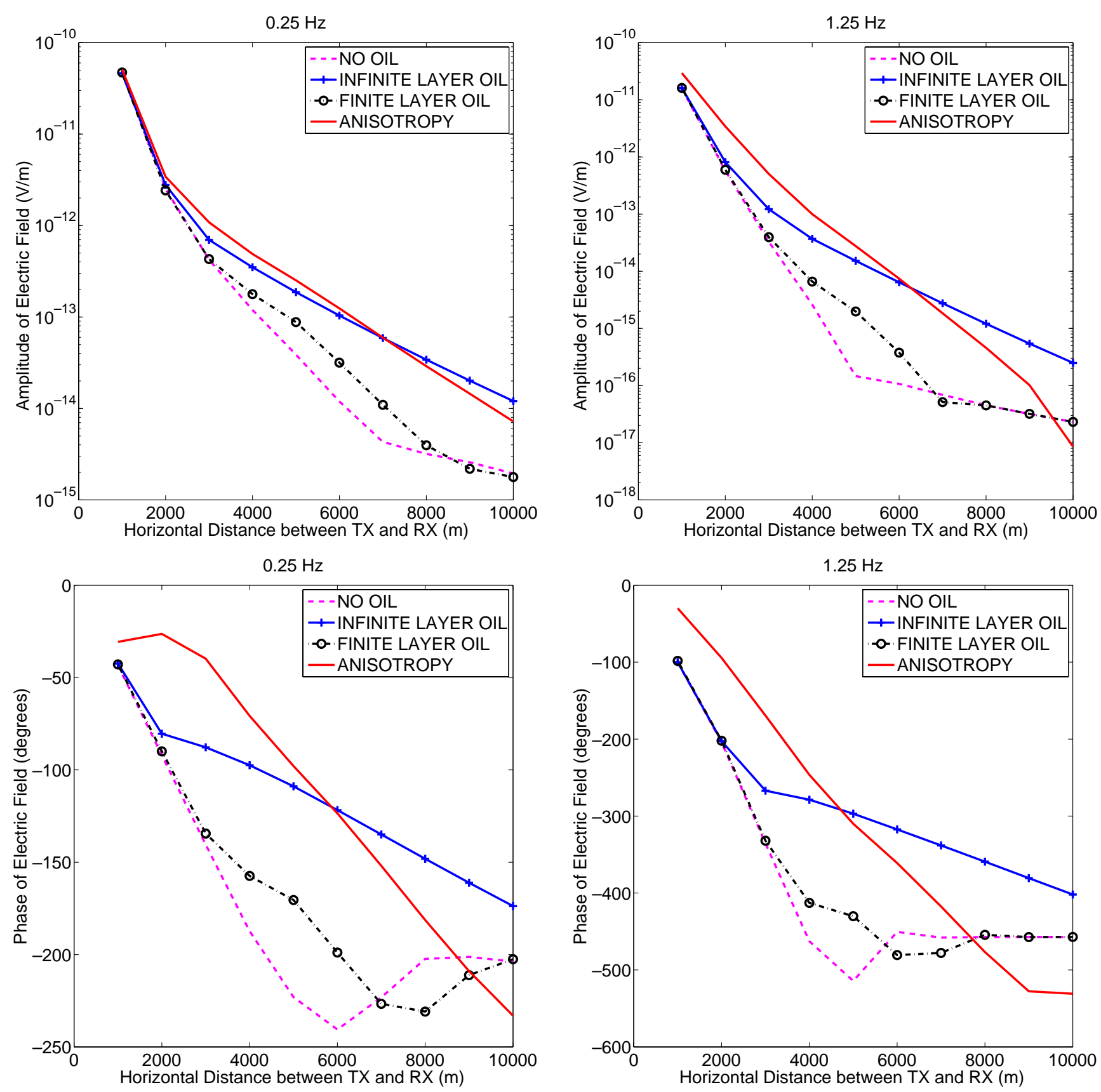

Fig. 9 Amplitude (top panel) and phase (bottom panel) of the electric field as a function of the horizontal distance between transmitter and receivers. Different curves indicate different models: a without oil (dotted pink), b with an infinite oil-bearing

layer (blue '+'), c with a oil-saturated finite layer (black circles), and $\mathbf{d}$ with a finite oil-bearing layer in an anisotropic formation. Operating frequencies: $0.25 \mathrm{~Hz}$ (left panel) and $1.25 \mathrm{~Hz}$ (right panel)

measurements are highly affected by it. Second, measurements obtained in the presence of an infinite oilbearing layer are quite different from those obtained in a presence of a finite oil-bearing layer. Third, results are sensitive to the presence of the finite oil-bearing layer, and features such as its location and size can be derived from the measurements.

The relative difference as we include the finite oilbearing layer with respect to measurements without an oil-bearing layer are displayed in Fig. 10. These results illustrate the dependance of the measurements with respect to the finite extent oil-bearing layer. Large relative differences in the measurements are observed both for the amplitude and the phase. The largest differences 

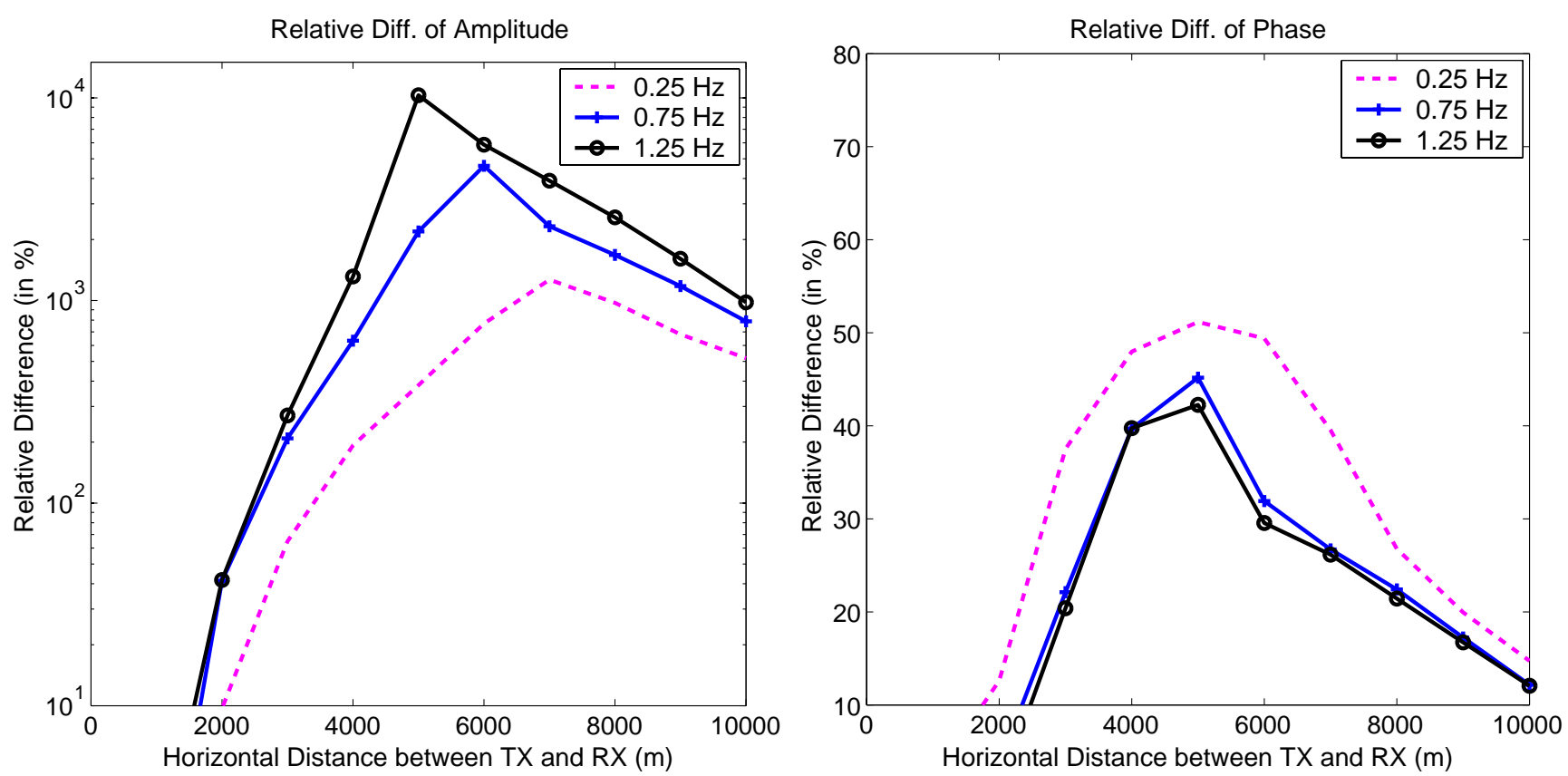

Fig. 10 Relative difference in (percentage) of the amplitude (left panel) and phase (right panel) of the results with a finite oil-bearing layer with respect to the case without an oil-bearing layer. Different curves correspond to various frequencies, from 0.25 to $1.25 \mathrm{~Hz}$

are localized around the location of the oil-bearing layer.

All problems described in this Section have been solved on a computer equipped with $32 \mathrm{~GB}$ of RAM and using only one core of the available $2 \mathrm{GHz}$ dualcore processor. The amount of time needed for computations never exceeded half an hour per receiver (in most cases only a few minutes was sufficient). The highaccuracy and limited computational resources used by the method indicate its suitability for solving inverse marine CSEM problems (perhaps using the parallel version for the case of inverse problems).

\section{Conclusion}

We introduced a Fourier $h p$-Finite Element formulation for the simulation of marine CSEM measurements. This formulation combines the use of higher-order FEM with the use of small elements to efficiently approximate singularities. The formulation was implemented using efficient direct solvers of linear equations, a self-adaptive goal-oriented refinement strategy, and a parallel implementation. This technology enables highly accurate and efficient simulations of marine CSEM measurements in a very limited CPU time.

Simulation results were verified using simple models based on a typical CSEM acquisition system in order to illustrate the accuracy of the method. We showed numerically that very limited number of Fourier modes (typically between five and nine) is enough to deliver very accurate simulations.

Additional simulations were performed to study and illustrate the main physical properties inherent to marine CSEM measurements. In particular, we showed the need for modeling electrically anisotropic formations, and the ability of marine CSEM measurements to detect finite oil-bearing layers.

Acknowledgements We are thankful to Dr. K.H. Lee, for providing us with EM1D, a software used for computing the exact solution for our model marine CSEM problem.

\section{References}

1. Alumbaugh, D.L., Newman, G.A.: Image appraisal for 2D and 3D electromagnetic inversion. Geophysics 65, 1455-1467 (2000)

2. Arnold, D.N., Falk, R.S., Winther, R.: Multigrid in H(div) and H(curl). Numer. Math. 85(2), 197-217 (2000)

3. Cecot, W., Rachowicz, W., Demkowicz, L.: An $h p$-adaptive finite element method for electromagnetics. III: a threedimensional infinite element for Maxwell's equations. Int. J. Numer. Methods Eng. 57(7), 899-921 (2003)

4. Chave, A.D., Cox, C.S.: Controlled electromagnetic sources for measuring electrical conductivity beneath the oceans. 1 . Forward problem and model study. J. Geophys. Res. 87, 5327-5338 (1982) 
5. Christensen, N.B., Dodds, K.: 1D inversion and resolution analysis of marine CSEM data. Geophysics 72, WA27-WA38 (2007)

6. Constable, S., Srnka, L.J.: An introduction to marine controlled-source electromagnetic methods for hydrocarbon exploration. Geophysics 72, WA3-WA12 (2007)

7. Demkowicz, L.: Computing with $h p$-adaptive finite elements. In: One and Two Dimensional Elliptic and Maxwell Problems, vol. 1. Chapman and Hall (2006)

8. Demkowicz, L., Kurtz, J., Pardo, D., Paszynski, M., Rachowicz, W., Zdunek, A.: Computing with hp-adaptive finite elements. In: Frontiers: Three-Dimensional Elliptic and Maxwell Problems with Applications, vol. 2. Chapman and Hall (2007)

9. Edwards, N.: Marine controlled source electromagnetics: principles, methodologies, future commercial applications. Surv. Geophys. 26, 675-700 (2005)

10. Gomez-Revuelto, I., Garcia-Castillo, L.E., Pardo, D., Demkowicz, L.: A two-dimensional self-adaptive $h p$-finite element method for the analysis of open region problems in electromagnetics. IEEE Trans. Magn. 43(4), 1337-1340 (2007)

11. Gomez-Revuelto, I., Garcia-Castillo, L.E., Demkowicz, L.F.: A comparison between several mesh truncation methods for $h p$-adaptivity in electromagnetics. Invited paper to the special session "Numerical methods for solving maxwell equations in the frequency domain". Torino (Italia), September 2007

12. Gribenko, A., Zhdanov, M.: Rigorous 3D inversion of marine CSEM data based on the integral equation method. Geophysics 72, WA73-WA84 (2007)

13. Harrington, R.F.: Time-Harmonic Electromagnetic Fields. McGraw-Hill, New York (1961)

14. Hiptmair, R.: Multigrid method for Maxwell's equations. SIAM J. Numer. Anal. 36(1), 204-225 (1998)

15. Hoversten, G.M., Chen, J., Gasperikova, E., Newman, G.A.: Integration of marine CSEM and seismic AVA data for reservoir parameter estimation. SEG/Houston Annual Meeting, EM 3.4, 579-583 (2005)

16. Hoversten, G.M., Newman, G.A., Flanagan, G.: 3D modeling of a deepwater EM exploration survey. Geophysics 71, G239G248 (2006)

17. Li, Y., Constable, S.: 2D marine controlled-source electromagnetic modeling: part 2, the effect of bathymetry. Geophysics 72, WA63-WA71 (2007)

18. Li, Y., Key, K.: 2D marine controlled-source electromagnetic modeling: part 1, an adaptive finite-element algorithm. Geophysics 72, WA51-WA62 (2007)

19. METIS: family of multilevel partitioning algorithms. http:// glaros.dtc.umn.edu/gkhome/views/metis (2007). Accessed 30 June 2010

20. MUMPS: A multifrontal massively parallel sparse direct solver (2010)
21. Newman, G.A., Commer, M., Carazzone, J.J.: Imaging CSEM data in the presence of electrical anisotropy. Geophysics 75, F51-F61 (2010)

22. Orange, A., Key, K., Constable, S.: The feasibility of reservoir monitoring using time-lapse marine CSEM. Geophysics 74, F21-F29 (2009)

23. PARDISO: Thread-safe solver of linear equations. http:// www.pardiso-project.org/ (2008). Accessed 30 June 2010

24. Pardo, D., Demkowicz, L., Torres-Verdín, C., Michler, C.: PML enhanced with a self-adaptive goal-oriented hp finiteelement method and applications to through-casing borehole resistivity measurements. SIAM J. Sci. Comput. 30, 2948 2964 (2008)

25. Pardo, D., Demkowicz, L., Torres-Verdín, C., Paszynski, M.: Simulation of resistivity logging-while-drilling (LWD) measurements using a self-adaptive goal-oriented $h p$-finite element method. SIAM J. Appl. Math. 66, 2085-2106 (2006)

26. Pardo, D., Demkowicz, L., Torres-Verdín, C., Paszynski, M.: A goal oriented $h p$-adaptive finite element strategy with electromagnetic applications. Part II: electrodynamics. Comput. Methods Appl. Mech. Eng. 196, 3585-3597 (2007)

27. Pardo, D., Demkowicz, L., Torres-Verdín, C., Tabarovsky, L.: A goal-oriented $h p$-adaptive finite element method with electromagnetic applications. Part I: electrostatics. Int. J. Numer. Methods Eng. 65, 1269-1309 (2006)

28. Pardo, D., Torres-Verdín, C., Demkowicz, L.: Simulation of multi-frequency borehole resistivity measurements through metal casing using a goal-oriented $h p$-finite element method. IEEE Trans. Geosci. Remote Sens. 44, 2125-2135 (2006)

29. Pardo, D., Torres-Verdín, C., Demkowicz, L.: Feasibility study for two-dimensional frequency dependent electromagnetic sensing through casing. Geophysics 72, F111-F118 (2007)

30. Pardo, D., Torres-Verdín, C., Nam, M.J., Paszynski, M., Calo, V.M.: Fourier series expansion in a non-orthogonal system of coordinates for simulation of $3 \mathrm{D}$ alternating current borehole resistivity measurements. Comput. Methods Appl. Mech. Eng. 197, 3836-3849 (2008)

31. Paszynski, M., Kurtz, J., Demkowicz, L.: Parallel fully automatic hp-adaptive 2D finite element package. Comput. Methods Appl. Mech. Eng. 195(7-8), 711-745 (2006)

32. Song, Y., Kim, H.J., Lee, K.H.: High frequency electromagnetic impedance for subsurface imaging. Geophysics 67 , 501-510 (2002)

33. Tompkins, M.J., Srnka, L.J.: Marine controlled-source electromagnetic methods: introduction. Geophysics 72, WA1WA2 (2007)

34. Um, E.S., Alumbaugh, D.L.: On the physics of the marine controlled-source electromagnetic method. Geophysics 72, WA13-WA26 (2007) 